\title{
GATA-2 mutation: An emerging syndrome
}

\author{
Lalit Raut
}

Dept of Medicine, Leukemia/BMT division, Vancouver General Hospital, Vancouver, Canada

\section{Email address:}

lalitraut76@yahoo.co.in and lalitraut1976@gmail.com

\section{To cite this article:}

Lalit Raut. GATA-2 Mutation: An Emerging Syndrome. Cancer Research Journal. Vol. 2, No. 4, 2014, pp. 70-73. doi: $10.11648 /$ j.crj.20140204.12

\begin{abstract}
Different clinical conditions of immunodeficiency and leukemic transformation were described recently. These are being associated with GATA-2 mutations. This is emerging as a new and interesting syndrome. This is a review of this condition.
\end{abstract}

Keywords: GATA-2, MonoMAC Syndrome, Emberger Syndrome, DCML Deficiency Syndrome, Familial MDS, Myelodysplastic Syndrome

\section{Introduction}

'The process of scientific discovery is, in effect, a continual flight from wonder.'

- Albert Einstein

The continuous hunger for knowledge leads to interesting discoveries. Medical field have seen several breath holding discoveries in the past and new discoveries continue to thrill us. The detection of GATA-2 mutations is one such example. The role of GATA transcription factors in carcinogenesis is well established. Mutations in GATA1 are linked to megakaryoblastic leukemias in Down syndrome; loss of GATA3 expression to aggressive, dedifferentiated breast cancers; and silencing of GATA4 and GATA5 expression to colorectal and lung cancers.(1) GATA-2 plays a critical role in both hematopoietic stem cell development and the maintenance of normal adult stem cell homeostasis. It is thought that GATA-2 mutations association with conditions like Emberger syndrome, MonoMAC syndrome, familial myelodysplastic syndrome, dendritic cell, monocyte, B- and NK-cell (DCML) deficiency is going to change the way we manage these conditions. This article is an attempt to explore the emerging field of conditions associated with GATA-2 mutations.

\section{GATA-2}

GATA motif is a cis-regulatory element of diverse genes that participate in transcription. It first became apparent in erythroid cells but was later found to have expression in other haemopoietic lineage and endothelial cells.(2)
GATA binding proteins are nuclear proteins to which cis-regulatory elements bind and modulate their activity. The GATA family has 6 proteins labeled as GATA-1, GATA-2 and so on. These proteins have a characteristic pattern of expression during development. They have distinctive and novel structure resembling zinc finger of cys-cys variety. GATA-1,2,3 differ in terms of selected amino acid sequences.(2) The expression of the first 3 GATA proteins are summarised in table 1.(3) The different mice studies suggest possible role of GATA-2 in haematopoiesis, neuronal development and transcriptional control of several genes expressed in endothelium. Moreover, it controls apoptosis.(4) The clinical syndromes with GATA-2 mutation suggest its probable role in lymphatics and ear development. Thus, GATA family proteins control broad developmental process.

GATA-2 is expressed in erythroid and other haematopoietic progenitors and its expression reduces as the level of GATA-1 increases with erythroid differentiation. The forced expression of GATA-2 promotes proliferation and blocks differentiation. Thus, GATA-2 is essential for appropriate expression of early haematopoietic cells.(5)

The $14 \mathrm{~kb}$ gene of GATA-2 gene is located on chromosome 3 . Broadly three types of GATA-2 mutations are described (i) N-terminal frameshift mutations resulting in premature termination of GATA-2 protein translation, and (ii) mutations in the first and second zinc fingers of GATA-2 and iii) regulatory mutations within the enhancer region of intron 5. Each mutation confers differing 
transcriptional activities probably contributing to variations in different syndromes.

GATA-2 overexpression was reported as a poor prognostic marker in acute myeloid leukemia (AML).(6) In a subset of chronic myeloid leukemia (CML) patients it was shown that GATA-2 mutations play role in myeloid blastic transformation.(7) Recently, GATA-2 mutations were associated with syndromes like MonoMAC syndrome, Emberger syndrome, DCML deficiency syndrome and Familial MDS.(8)(9)(10)(11) There is variation in the characteristics of these diseases and they are progressive in nature. The risk of progression to MDS/AML varies with each group. These conditions are described in the following paragraphs.

\section{Mono MAC Syndrome}

The name of MonoMAC syndrome is derived from monocytopenia and nontuberculous mycobacterial infection. MonoMAC syndrome was associated with GATA-2 mutation in 2011.(8) It is characterized by disseminated nontuberculous mycobacterial and other opportunistic infections. Moreover, it is also associated with an increased incidence of myelodysplasia and malignancy. It is inherited as autosomal dominant disease or could be sporadic. It is commonly observed in adulthood. Recurrent or disseminated viral and fungal infections are also frequent. Persistent monocytopenia and NK lymphopenia is a characteristic feature and may precede other clinical manifestations. Low CD4, CD8 count and neutropenia may be seen. Inspite of marked B lymphocytopenia immunoglobulin levels are normal. GM-CSF antibody negative pulmonary alveolar proteinosis (PAP) is also a classical complication in addition to predisposition to myelodysplasia and AML.(12) See table 2 for classical features of MonoMAC syndrome.

Myelodysplasia in MonoMAC syndrome: Calvo et al. analysed bone marrow findings of 18 patients with MonoMAC syndrome.(13) These patients often have first degree relatives with history of opportunistic infections, MDS or leukemia. Hypocellularity, trilineage dysplasia, fibrosis, abnormal plasma cells and presence of histiocytes are frequently found than in denovo MDS cases. The evolution to MDS is seen in 3rd or 4th decade of life. Thus, myelodysplasia associated with MonoMAC syndrome is different from denovo MDS and other inherited bone marrow syndromes.

MonoMac versus Idiopathic CD4 Lymphocytopenia(ICL): It is common to confuse MonoMAC with ICL especially in the early stage of the disease. History of opportunistic infections, autoimmune phenomena and profound NK and B cell lymphocytopenia are common findings. In MonoMAC syndrome, both the CD4 and CD8 count is low whereas in ICL though CD4 count is less the CD4:CD8 ratio is reversed.(14) Monocytopenia, classical bone marrow findings and GATA-2 mutations are commonly associated with MonoMAC syndrome.
DCML deficiency is same as MonoMAC syndrome except for the MAC infection. (15)

\section{Emberger Syndrome}

This syndrome is named after Emberger who first described individuals from two generations with lymphedema, haematological abnormalities and deafness.(16) The condition is probably inherited as autosomal dominant or sporadic pattern. The cardinal feature is childhood onset of unilateral or bilateral primary lymphedema of lower limbs. Genital or intestinal lymphatic abnormalities are also found in few patients. Lymphoscintigraphy demonstrates lymphatic vessel hypoplasia. They have predisposition to develop myelodysplasia/AML characteristically associated with monosomy 7. Other minor anomalies include deafness, warts, abnormalities in the lymphocyte subsets and skeletal abnormalities like hypotelorism.(17) Key for the diagnosis is to exclude conditions like Noonan syndrome with juvenile myelomonocytic leukemia (JMML) and familial MDS. The association of haematological abnormalities and primary lymphedema could be linked to the fact that hemangioblast gives rise, during development, to both haemopoietic stem cells and angioblasts from which lymphatics and other blood vessels develop.

Haploinsufficiency of GATA2 was linked with Emberger syndrome.(9) Kazenwadel et al. illustrated that several of the key genes expressed highly in, and important for, the construction of lymphatic valves are regulated by GATA2. Failure of these valves to form or function to full capacity affects the maturity of lymphatics resulting in lymphedema. (18)

GATA2 is also important in vestibular morphogenesis and growth of semicircular canals.(19) This could explain the sensorineural deafness in Emberger syndrome. But the reason for its presence in only some patients is not known.

\section{Familial MDS/AML}

The MDS are a heterogeneous group of clonal haematopoietic stem cell disorders characterized by dysplastic changes and ineffective haematopoiesis leading to cytopenias and risk of evolution into AML. MDS/AML is labelled as familial if an AML/MDS patient's family has two or more members with bone marrow failure syndromes. Familial MDS/AML is linked to mutations in RUNX1, CEBPA, TERT, TERC etcetera. GATA-2 mutation was discovered as a predisposing factor in the development of familial MDS/AML.(11) Ishida et al. described GATA-2 mutation in a patient with clinical features common with Emberger, MonoMAC and DCML deficiency.(20) This suggests that probably these syndromes are different phenotypes caused by genetic alteration in GATA-2. 


\section{GATA-2 Mutation}

GATA-2 mutation produces a phenotype with abnormality of hematopoiesis, immunity, lymphatics, pulmonary function and risk of MDS/AML. As discussed before, the mutation could be sporadic or inherited in an autosomal dominant pattern and presents clinically in early childhood to late adulthood. There is no gender or race predisposition. The mutation could be found in completely asymptomatic individuals through family screening. The most common initial presentation is infections like severe viral, mycobacterial and fungal infections. MDS/AML, lymphedema are some of the other presenting features. The common haematological findings are B lymphocytopenia, NK lymphocytopenia, monocytopenia, pancytopenia, myelodysplasia, trisomy 8 , monosomy 7 , AML and chronic myelomonocytic leukemia (CMML). These patients can present with recurrent warts, panniculitis and sweet syndrome. Skin cancers like squamous cell carcinoma, basal cell carcinoma, malignant melanoma; Human papilloma virus related dysplasia; Epstein Barr Virus positive leiomyosarcomata and many other solid tumors are also reported. Deep vein thrombosis, pulmonary embolism, hepatic vein thrombosis and catheter related thrombosis are important observations. The thrombotic abnormalities are probably caused by endothelial abnormalities due to GATA-2 mutations. Sensorineural deafness, idiopathic hypothyroidism, recurrent miscarriages are other associated clinical features.(21)

GATA-2 mutation leads to allele haploinsufficiency. The expression of hemopoietic precursors and their number gradually reduces. GATA-2 mutation probably results in endothelial, trophoblastic giant cell dysfunction and abnormality in lymphatic as well as otic development. GATA-2 mutation affects beta thyrotropin function leading to hypothyroidism. The mechanism underlying these developments is yet to be discovered. Thus, the pathophysiology of this condition is poorly understood and so is the genotype-phenotype correlation.

Analysis of several patients described with GATA-2 mutation suggests clinical heterogeneity ranging from completely asymptomatic state to full blown malignancies. The evolution from asymptomatic phase of cellular deficiency, acquisition of cytogenetic changes, development of cytopenia and progression to MDS/AML is best explained by the model given by Venetia Bigley and Matthew Collin.(22) In search for the reason of this varied clinical presentation scientists studied genotypes of GATA-2 mutation but found no genotype-phenotype correlation except for the occurrence of lymphedema. According to Kazenwadel et al. incomplete penetrance of the lymphedema phenotype could be due to retention of partial activity of missense mutations of GATA2, which renders them less disruptive to lymphatic vascular function than heterozygous GATA2 deletion.(18) Clearly, GATA-2 mutation could be the disease initiating event but further insults from second hits like environmental factors probably modifies the clinical presentation and needs to be studied.

Early diagnosis is the key in the management of these patients. It is difficult to recommend which patients should be tested for GATA-2 mutation but the clinical features could be able to provide a rough guidance. The mutation is detected by sequencing studies from genomic DNA obtained from peripheral blood, buccal swabs or fibroblasts. All family members should be tested. Prevention of infections by prophylactic antibiotics, antivirals; annual screening for malignancies and monitoring organ functions is very important. Often the infections are difficult to treat. The severity of cytopenias and development of complications like MDS/AML decide the management strategy in others. The age wise overall survival (OS) rate noticed by Spinner et al. was $96 \%$ by age 20 years, $77 \%$ by age 40 years, and $45 \%$ by age 60 years. The proportion of patients surviving without hemopoietic stem cell transplant (HSCT) reported by the same group was $89 \%$ by age 20 years, $76 \%$ by age 30 years, and $53 \%$ by age 40 years. Stem cell transplant, the only curative option is indicated for MDS/AML, recurrent infection and PAP. In the same study, the posttransplant survival rate was reported as $72 \%$ by 1 year, $65 \%$ by 2 years, and $54 \%$ by 4 years after HSCT.(21)

Thus, GATA-2 mutation leads to interesting and varied clinical manifestations. Many questions need to be explored about this emergent syndrome. With the increase in awareness, early diagnosis, improved surveillance for malignancies and growing experience in HSCT the survival of patients with GATA-2 mutation is surely going to improve in future.

\section{Future Aspects}

Further studies are required:

- for better clinical characterization of patients with GATA-2 mutation

- for understanding the underlying pathogenesis of this condition

- $\quad$ to delineate the molecular and cellular mechanisms that contribute to the variable phenotypic expression of this disorder.

- $\quad$ to search for targeted drug therapy

\section{References}

[1] Zheng R, Blobel GA. GATA Transcription Factors and Cancer. Genes Cancer. 2010 Dec;1(12):1178-88.

[2] Orkin SH. GATA-binding transcription factors in hematopoietic cells. Blood. 1992 Aug 1;80(3):575-81.

[3] Simon MC. Gotta have GATA. Nat Genet. 1995 Sep;11(1):9-11.

[4] Rodrigues NP, Janzen V, Forkert R, Dombkowski DM, Boyd AS, Orkin SH, et al. Haploinsufficiency of GATA-2 perturbs adult hematopoietic stem-cell homeostasis. Blood. $2005 \mathrm{Jul}$ 15;106(2):477-84. 
[5] Shivdasani RA, Orkin SH. The transcriptional control of hematopoiesis [see comments]. Blood. 1996 May 15;87(10):4025-39.

[6] Vicente C, Vazquez I, Conchillo A, García-Sánchez MA, Marcotegui N, Fuster O, et al. Overexpression of GATA2 predicts an adverse prognosis for patients with acute myeloid leukemia and it is associated with distinct molecular abnormalities. Leukemia. 2012 Mar;26(3):550-4.

[7] Zhang S-J, Ma L-Y, Huang Q-H, Li G, Gu B-W, Gao X-D, et al. Gain-of-function mutation of GATA-2 in acute myeloid transformation of chronic myeloid leukemia. Proc Natl Acad Sci U S A. 2008 Feb 12;105(6):2076-81.

[8] Hsu AP, Sampaio EP, Khan J, Calvo KR, Lemieux JE, Patel $\mathrm{SY}$, et al. Mutations in GATA2 are associated with the autosomal dominant and sporadic monocytopenia and mycobacterial infection (MonoMAC) syndrome. Blood. 2011 Sep 8;118(10):2653-5.

[9] Ostergaard P, Simpson MA, Connell FC, Steward CG, Brice G, Woollard WJ, et al. Mutations in GATA2 cause primary lymphedema associated with a predisposition to acute myeloid leukemia (Emberger syndrome). Nat Genet. 2011 Oct;43(10):929-31.

[10] Dickinson RE, Griffin H, Bigley V, Reynard LN, Hussain R, Haniffa $M$, et al. Exome sequencing identifies GATA-2 mutation as the cause of dendritic cell, monocyte, B and NK lymphoid deficiency. Blood. 2011 Sep 8;118(10):2656-8.

[11] Hahn CN, Chong C-E, Carmichael CL, Wilkins EJ, Brautigan PJ, Li X-C, et al. Heritable GATA2 mutations associated with familial myelodysplastic syndrome and acute myeloid leukemia. Nat Genet. 2011 Oct;43(10):1012-7.

[12] Vinh DC, Patel SY, Uzel G, Anderson VL, Freeman AF, Olivier KN, et al. Autosomal dominant and sporadic monocytopenia with susceptibility to mycobacteria, fungi, papillomaviruses, and myelodysplasia. Blood. $2010 \mathrm{Feb}$ $25 ; 115(8): 1519-29$.

[13] Calvo KR, Vinh DC, Maric I, Wang W, Noel P, Stetler-Stevenson M, et al. Myelodysplasia in autosomal dominant and sporadic monocytopenia immunodeficiency syndrome: diagnostic features and clinical implications. Haematologica. 2011 Aug 1;96(8):1221-5.

[14] Zonios DI, Falloon J, Bennett JE, Shaw PA, Chaitt D, Baseler MW, et al. Idiopathic CD4+ lymphocytopenia: natural history and prognostic factors. Blood. 2008 Jul 15;112(2):287-94.
[15] Ishida H, Imai K, Honma K, Tamura S-I, Imamura T, Ito M, et al. GATA-2 anomaly and clinical phenotype of a sporadic case of lymphedema, dendritic cell, monocyte, B- and NK-cell (DCML) deficiency, and myelodysplasia. Eur J Pediatr. 2012 Aug;171(8):1273-6.

[16] Emberger JM, Navarro M, Dejean M, Izarn P. [Deaf-mutism, lymphedema of the lower limbs and hematological abnormalities (acute leukemia, cytopenia) with autosomal dominant transmission]. J Génétique Hum. 1979 Oct;27(3):237-45.

[17] Mansour S, Connell F, Steward C, Ostergaard P, Brice G, Smithson S, et al. Emberger syndrome-primary lymphedema with myelodysplasia: report of seven new cases. Am J Med Genet A. 2010 Sep;152A(9):2287-96.

[18] Kazenwadel J, Secker GA, Liu YJ, Rosenfeld JA, Wildin RS, Cuellar-Rodriguez J, et al. Loss-of-function germline GATA2 mutations in patients with MDS/AML or MonoMAC syndrome and primary lymphedema reveal a key role for GATA2 in the lymphatic vasculature. Blood. 2012 Feb 2;119(5):1283-91.

[19] Haugas M, Lilleväli K, Hakanen J, Salminen M. Gata2 is required for the development of inner ear semicircular ducts and the surrounding perilymphatic space. Dev Dyn Off Publ Am Assoc Anat. 2010 Sep;239(9):2452-69.

[20] Ishida H, Imai K, Honma K, Tamura S-I, Imamura T, Ito M, et al. GATA-2 anomaly and clinical phenotype of a sporadic case of lymphedema, dendritic cell, monocyte, B- and NK-cell (DCML) deficiency, and myelodysplasia. Eur J Pediatr. 2012 Aug;171(8):1273-6.

[21] Spinner MA, Sanchez LA, Hsu AP, Shaw PA, Zerbe CS, Calvo KR, et al. GATA2 deficiency: a protean disorder of hematopoiesis, lymphatics, and immunity. Blood. 2014 Feb 6;123(6):809-21

[22] Bigley V, Collin M. Dendritic cell, monocyte, B and NK lymphoid deficiency defines the lost lineages of a new GATA-2 dependent myelodysplastic syndrome. Haematologica. 2011 Aug 1;96(8):1081-3.

[23] Camargo JF, Lobo SA, Hsu AP, Zerbe CS, Wormser GP, Holland SM. MonoMAC Syndrome in a Patient With a GATA2 Mutation: Case Report and Review of the Literature. Clin Infect Dis. 2013 Sep 1;57(5):697-9. 